\title{
Bioprosthetic Valve Streptococcus bovis Endocarditis Secondary to Colon Cancer Presenting with a Lacunar Stroke
}

\author{
Benjamin Chin-Yee, Kalpa Shah,, Ori Rotstein,,3 Amro Nagy, Cameron Williams, \\ Howard Leong-Poi, ${ }^{3,4}$ Jonathan C. Lu, and Fahad Razak ${ }^{1,5,6}$
}

\begin{abstract}
Background: Streptococcus bovis endocarditis is an uncommon but reported complication of colon cancer. Studies show that approximately $50 \%$ of patients with S. bovis infection have underlying colonic neoplasm.

Case Presentation: An 82-year-old woman presented to the hospital with a lacunar stroke resulting from S. bovis endocarditis of her bioprosthetic aortic valve, ultimately leading to the diagnosis of colonic malignant disease. The patient's neurologic deficits resolved and she completed a course of intravenous antibiotics followed by a successful right hemicolectomy to remove the colonic neoplasm. We review recent literature on the association between $S$. bovis endocarditis and colon cancer and discuss the implications for the clinical management of this condition.

Conclusion: Because of the high rate of association between S. bovis infection and colonic neoplasms, the presence of $S$. bovis bacteremia should prompt a work-up for occult malignant disease by colonoscopy.
\end{abstract}

$S$ TREPTOCOCCUS BOVIS ENDOCARDITIS is an uncommon but reported complication of colon cancer. Studies suggest that approximately $50 \%$ of patients with $S$. bovis infection have underlying colonic neoplasm [1]. The pathobiology of this association is poorly understood, but may involve mucosal and vascular disruption resulting in increased translocation of enteric bacteria into the bloodstream. In this article we present a case of colon cancer presenting with a lacunar stroke secondary to $S$. bovis endocarditis. The patient underwent a successful right hemicolectomy for the colonic neoplasm and completed a course of intravenous antibiotics with resolution of neurologic deficits.

\section{Case Presentation}

An 82-year-old woman presented to the hospital with acute onset left-sided weakness and dysarthria. Past medical his- tory was significant for coronary artery disease and severe aortic stenosis. The previous year she had undergone coronary artery bypass grafting and bioprosthetic aortic valve replacement (Sorin Perceval ${ }^{\mathrm{TM}}$ sutureless valve; Sorin Group, Arvada, CO). The patient also had a history of paroxysmal atrial fibrillation and was previously on anti-coagulant therapy, however, this had been discontinued recently because of the development of an unexplained microcytic anemia. Six months prior to her presentation the patient began experiencing general malaise, anorexia, subjective fevers, and weight loss of $9 \mathrm{~kg}$.

On presentation, the patient was hemodynamically stable (blood pressure $112 / 78 \mathrm{~mm} \mathrm{Hg}$, heart rate 85 beats per minute) with a low-grade fever (temperature, $38.2^{\circ} \mathrm{C}$ ). Neurologic examination revealed left-sided weakness and facial droop. Laboratory investigations showed a hemoglobin of $76 \mathrm{~g} / \mathrm{L}$, mean corpuscular volume $68.7 \mathrm{fL}$, ferritin $20 \mathrm{mcg} / \mathrm{L}$,

\footnotetext{
${ }^{1}$ Division of General Internal Medicine, ${ }^{2}$ Division of General Surgery, ${ }^{4}$ Division of Cardiology, Faculty of Medicine, University of Toronto, Toronto, Ontario, Canada.

${ }^{3}$ Keenan Research Centre for Biomedical Science of St Michael's Hospital, ${ }^{5}$ Li Ka Shing Knowledge Institute of St Michael's Hospital, Toronto, Ontario, Canada.

${ }^{6}$ Harvard Centre for Population and Development Studies, Harvard University, Cambridge, Massachusetts.

(C) Benjamin Chin-Yee et al. 2016; Published by Mary Ann Liebert, Inc. This Open Access article is distributed under the terms of the Creative Commons License (http://creativecommons.org/licenses/by/4.0), which permits unrestricted use, distribution, and reproduction in any medium, provided the original work is properly credited.
} 
neutrophils $12.9 \times 10^{9} / \mathrm{L}$, and erythrocyte sedimentation rate (ESR) $40 \mathrm{~mm} / \mathrm{h}$. Magnetic resonance imaging of the head revealed multiple bilateral hemispheric infarcts with involvement of the right corona radiata (Fig. 1). Blood cultures drawn on admission grew $S$. bovis. Initial transthoracic echocardiography showed a well-seated bioprosthetic aortic valve with trace intra-valvular aortic regurgitation but was otherwise normal. Subsequent transesophageal echocardiography demonstrated a $0.7 \times 0.4 \mathrm{~cm}$ vegetation attached to the posterior aspect of the bioprosthetic aortic valve annulus with severely thickened valve cusps, consistent with infective endocarditis (Fig. 2). Colonoscopy was performed, which revealed a large ulcerated cecal mass; biopsies confirmed invasive adenocarcinoma. Staging computed tomography scan showed a mass involving the cecum and proximal ascending colon with localized extension into the distal ileum but no evidence of distal metastases (Fig. 3). Carcinoembryonic antigen (CEA) was $14 \mathrm{mcg} / \mathrm{L}$. On the basis of these findings the patient was diagnosed with a lacunar stroke secondary to $S$. bovis endocarditis, which was ultimately the result of an underlying colonic malignant disease.

The patient's weakness and dysarthria resolved during her admission, and she had no residual neurologic deficits. She was treated with a six-week course of intravenous ceftriaxone. Subsequent blood cultures were negative. Repeat transthoracic echocardiography again showed trace aortic regurgitation but no evidence of vegetation. The decision was made to perform a laparoscopic right hemicolectomy following the course of antibiotics. The operation was without complication and the patient was

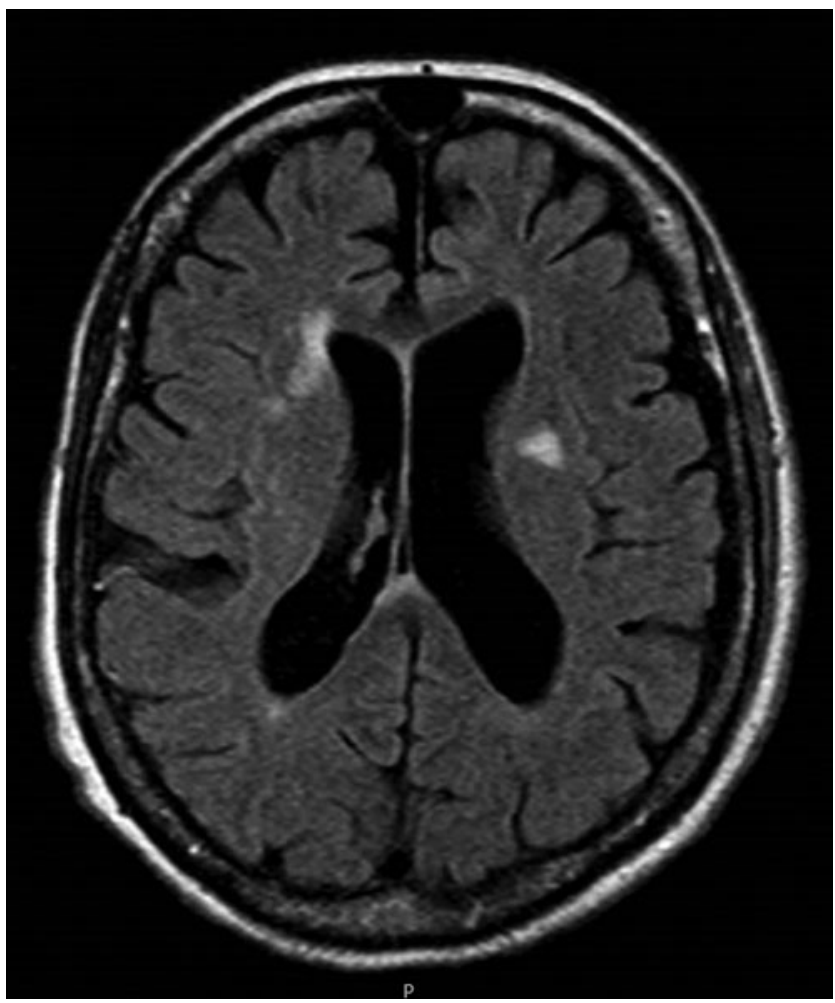

FIG. 1. Magnetic resonance imaging (MRI) T2-weighted fluid-attenuated inversion recovery (T2/FLAIR) showing multiple bilateral cerebral infarcts with involvement of the right corona radiata.

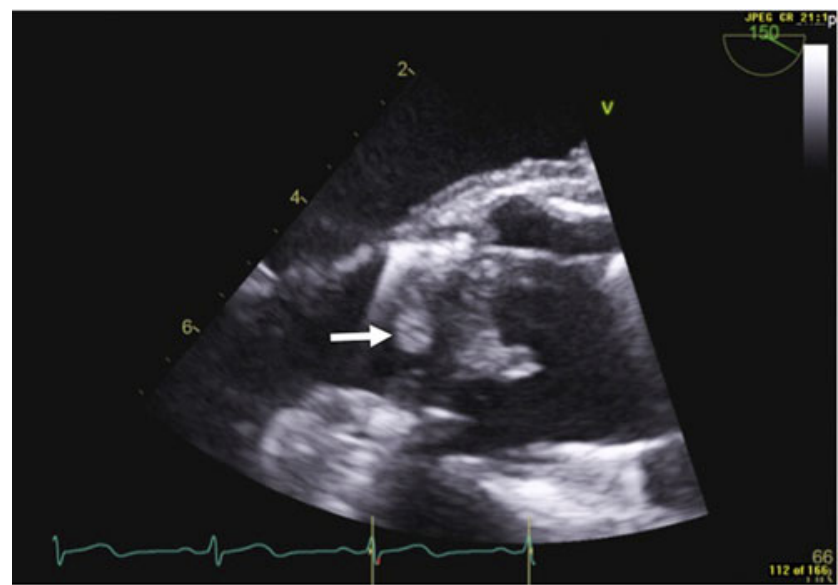

FIG. 2. Transesophageal echocardiography showing a $0.7 \times 0.4 \mathrm{~cm}$ vegetation attached to the posterior aspect of the bioprosthetic aortic valve annulus with severely thickened valve cusps, consistent with infective endocarditis.

discharged home. Histologic examination showed invasive adenocarcinoma through the muscular propria into the subserosa with negative resection margins. One of $25 \mathrm{lymph}$ nodes was positive for malignant disease, pT3pN1 [2]. The patient reported feeling well at follow-up. Repeat CEA was $1.7 \mathrm{mcg} / \mathrm{L}$. She is planning to start adjuvant chemotherapy with capecitabine.

\section{Discussion}

The link between $S$. bovis infection and colonic neoplasm is well described in the literature. Since the first case report by McCoy and Masson in 1951 [3], several studies have confirmed this association and investigated its underlying mechanism. Although the strength of this association varies with $S$. bovis subspecies as well as temporal and geographic factors, a recent meta-analysis estimates that approximately $50 \%$ of patients with $S$. bovis infection have concurrent colorectal neoplasm $[1,4]$.

Knowledge of the pathobiology of $S$. bovis infection in colonic malignant disease remains incomplete. Streptococcus

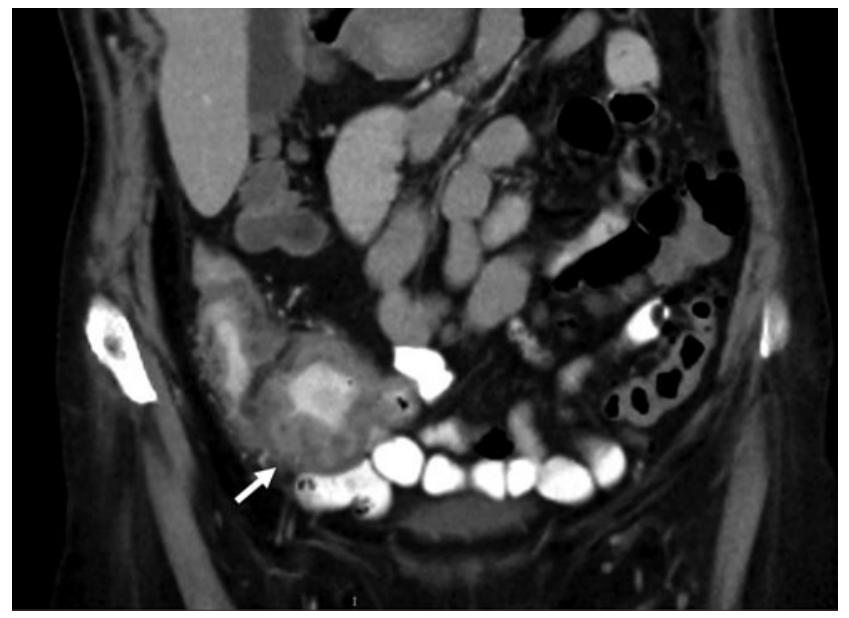

FIG. 3. Computed tomography of the abdomen, coronal view, showing $10-\mathrm{cm}$ mass involving the cecum and proximal ascending colon with localized extension into the distal ileum. 
bovis is a part of the normal intestinal flora in human beings. Streptococcus bovis bacteremia is believed to result from increased bacterial translocation into the bloodstream, facilitated by the affinity of $S$. bovis for extracellular matrix proteins exposed during tumor growth and tissue injury [5]. Some preclinical studies, however, have suggested that $S$. bovis may play a causal role in the development of colonic neoplasms, promoting oncogenesis by induction of cytokines via bacterial cell wall antigens [6]. Although these data remain controversial, based on the strength of the association, some authors have proposed that $S$. bovis-related biomarkers may find applications in screening for colorectal cancer [7].

Our case emphasizes several salient points in the diagnosis and management of patients with $S$. bovis endocarditis and associated colon cancer. Regarding diagnosis, this condition can have a varied presentation. In a patient presenting with stroke-especially one related to embolic phenomena-and microcytic anemia, one should consider occult malignant disease as the unifying diagnosis. Furthermore, the presence of $S$. bovis bacteremia should always prompt a work-up for colonic neoplasm by colonoscopy [8,9]. Despite the wellrecognized association between $S$. bovis infection and colon cancer, unfortunately not all patients undergo appropriate investigations, which should be performed as an inpatient if adequate follow-up cannot be guaranteed [8]. The $2015 \mathrm{Eu}-$ ropean Society of Cardiology guidelines for the management of infective endocarditis support this recommendation, and further suggest follow-up with annual colonoscopy if initial investigations are negative [9].

Regarding management, one challenge encountered in this case was deciding the timing of surgery after the patient's presentation with stroke and subsequent diagnosis of colon cancer. There is a paucity of evidence to support an optimal timing of surgery after stroke, and we are unaware of any studies examining this question specifically in the context of cardioembolic strokes secondary to infective endocarditis. Based on interdisciplinary discussions between medical and surgical teams, the decision was made to delay surgery until the completion of a sixweek course of antibiotics, after which time a laparoscopic right hemicolectomy was performed. The overall outcome was favorable: the patient tolerated the surgery well and experienced no complications.

\section{Conclusions}

This case describes an uncommon presentation of a common disease: colon cancer presenting with a lacunar stroke secondary to $S$. bovis endocarditis. It emphasizes further the importance of performing colonoscopy in all patients with S. bovis bacteremia to diagnose a potentially concurrent colorectal neoplasm and allow for appropriate management. This case also raises the question of the optimal timing of surgery after stroke, an important topic in need of further study with a particular focus on elucidating different potential risk categories based on stroke etiology.

\section{Author Disclosure Statement}

No competing financial interests exist.

\section{References}

1. Krishnan S, Eslick GD. Streptococcus bovis infection and colorectal neoplasia: A meta-analysis. Colorectal Dis 2014; 16:672-680.

2. Frederick LG, Page DL, Fleming ID, et al. AJCC Cancer Staging Manual. New York, NY: Springer Science \& Business Media; 2013.

3. McCoy WC, Mason JM. Enterococcal endocarditis associated with carcinoma of the sigmoid: Report of a case. J Med Assoc State Ala 1951;21:162-166.

4. Beck M, Frodl R, Funke G. Comprehensive study of strains previously designated Streptococcus bovis consecutively isolated from human blood cultures and emended description of Streptococcus gallolyticus and Streptococcus infantarius subsp. coli. J Clin Microbiol 2008;46:2966-2972.

5. Sillanpää J, Nallapareddy SR, Singh KV, et al. Adherence characteristics of endocarditis-derived Streptococcus gallolyticus ssp. gallolyticus (Streptococcus bovis biotype I) isolates to host extracellular matrix proteins. FEMS Microbiol Lett 2008;289:104-109.

6. Ellmerich S, Schöller M, Duranton B, et al. Promotion of intestinal carcinogenesis by Streptococcus bovis. Carcinogenesis 2000;21:753-756.

7. Boleij A, van Gelder MMHJ, Swinkels DW, et al. Clinical importance of Streptococcus gallolyticus infection among colorectal cancer patients: Systematic review and meta-analysis. Clin Infect Dis 2011;53:870-878.

8. Ferrari A, Botrugno I, Bombelli E, et al. Colonoscopy is mandatory after Streptococcus bovis endocarditis: A lesson still not learned. World J Surg Oncol 2008;6:49-45.

9. Habib G, Lancellotti P, Antunes MJ, et al. 2015 ESC Guidelines for the management of infective endocarditis. Eur Heart J 2015;36:3075-3128.

Address correspondence to: Dr. Benjamin Chin-Yee 152 St. Patrick Street, Unit 908 Toronto, Ontario M5T J39 Canada

E-mail: benjamin.chinyee@mail.utoronto.ca

$$
\begin{aligned}
& \text { Abbreviations Used } \\
\mathrm{ESR}= & \text { erythrocyte sedimentation rate } \\
\mathrm{CEA}= & \text { carcinoembryonic antigen } \\
\mathrm{MRI}= & \text { magnetic resonance imaging } \\
\mathrm{T} 2 / \mathrm{FLAIR}= & \mathrm{T} 2 \text {-weighted fluid-attenuated } \\
& \text { inversion recovery }
\end{aligned}
$$

Cite this article as: Chin-Yee $\mathrm{B}$, Shah $\mathrm{K}$, Rotstein $\mathrm{O}$, Nagy A, Williams C, Leong-Poi H, Lu JC, Razak F (2016) A case of bioprosthetic valve Streptococcus bovis endocarditis secondary to colon cancer presenting with a lacunar stroke. Surgical Infections Case Reports 1:1, 63-65, DOI: 10.1089/crsi.2016. 